\title{
Smart Home loT Sensors: Principles and Applications A Review of Low-Cost and Low-Power Solutions
}

\begin{abstract}
Mini Review
Volume 2 Issue 1- 2021

Author Details

Alexandros Gazis*, Eleftheria Katsiri

Department of Electrical and Computer Engineering, Democritus University of Thrace, Greece

*Corresponding author

Alexandros Gazis, Teaching Assistant, Department of Electrical and Computer Engineering, Democritus University of Thrace, Xanthi, Greece

Article History

Received: February 01, 2021 Accepted: February 15, 2021 Published: February 16, 2021

Abstract: Computer scientists and researchers have focused their efforts on advancing from traditional computing techniques to the use of "Industry 4.0". Specifically, this term includes breakthroughs in numerous fields, including manufacturing, Artificial Intelligence, machine learning and data science. Additionally, emphasis is placed on the so-called "Internet of Things", i.e. the development of interconnected devices capable of communicating and processing information, mainly from sensory networks. This article presents electronic devices used for home automation. Specifically, it presents different types of residential sensors which can transform a traditional house to a "smart home". Furthermore, it reviews their possible uses, such as for ventilation, security and temperature monitoring. Lastly, for each of the sensors, the article recommends low-cost sensory devices to set up affordable home automation projects, such as an Arduino and a Raspberry Pi.
\end{abstract}

Keywords: Smart homes, Smart houses, Smart sensors, Residential sensors, Wireless sensor networks, Low-Power sensors, Low-Cost sensors, Arduino, Raspberry Pi, Internet of things, Industry 4.0

\section{Introduction}

Recent studies suggest that many fields of computer science will be significantly affected by the so-called "Industry 4.0 ". This term is used to describe the fourth industrial revolution, which will make an extensive and fundamental difference to the industry. More specifically, it will connect humans with machines via the use of smart technologies. The term "smart" is used by scientists when they refer to the Internet of Things (IoT) and the Internet of People [1]. The first refers to large interconnected networks of computer devices (e.g. sensors) which can rapidly exchange vast amount of information. The second refers to the technical education that people will need to acquire to benefit from the endless capabilities of autonomous devices.

IoT is frequently used in cloud computing, cyber security, Artificial Intelligence, Big Data and analytics [2,3]. While it is not a panacea, it can introduce decentralized decision making, enhance information transparency, and augment interconnectivity between electronic devices, manufacturing and commerce procedures. Io T is by nature a system that heavily relies on data; indicatively more than 2.5 quintillion bytes of data are generated daily [4] and their number is increasing exponentially. A large portion of that data is - and will continue to be - generated from interconnected electronic devices (e.g., laptops, cellphones, smart TVs and smart vehicles) equipped with an abundance of sensory components $[5,6]$. In other words, there is so much technology involved in our everyday lives due to the use of devices. Globally, there are millions of electronic devices connected to the Internet, the majority of which require cellular or $\mathrm{Wi}$-Fi connections to operate. IoT must now overcome two challenges in order to further blossom: the insufficient number of interconnected sensors and limited access to sensory data $[7,8]$.

One of the projects using IoT which requires additional research is "smart homes", i.e., placing sensors to facilitate domestic life. Either to upgrade older houses or to enhance the capabilities of recent constructions, residential sensors are gradually integrated in the design of smart homes [9]. A smart home checks the installed infrastructure in order to group certain functions and automate others. In home automation, we can integrate air conditioning systems, smart lighting, CCTV and HVAC systems. This way, the homeowner can have overall control and supervision of a home locally, through wall switches, remote controls, or remotely via the internet. A smart home needs to 
allow us when we are indoors to act easily, without moving through different rooms to perform said tasks. When we are outdoors, it needs to allow us to act as if we were indoors. The owner is enabled to switch on the heating, unlock the door to a trusted visitor and generally act as if he/she was inside the premises.

Home automation architecture includes but is not restricted to:

- A central gateway, that coordinates home control

- Smart sockets, for energy management

- Various sensors and actuators

- A home area network (wireless, low power, star or mesh topology).

Moreover, in the market, there are many built-in, "batteries included" home automation solutions from various vendors, such as Samsung's SmartThings [10], Amazon's Alexa Cloud [11], Apple's Home Kit [12] and Google's Home Assistant [13]. Some of these systems support user interaction through a mobile app or a web page (Google Home, Apple Home, Mi Home), or by simple voice commands, or even natural language (Amazon Alexa, Apple Siri, Circe).

This article briefly presents applications regarding residential sensors and the most common sensory devices used to set up smart home automation systems. Specifically, the analysis is fourfold; it covers core sensor devices used in smart automation and showcases successful sensor-implemented home applications. Then, it shortly explains the operation procedure of the sensors discussed and suggests lowcost and low-power sensory components. These suggestions can be adopted to develop smart homes, using inexpensive micro-controllers and mini-computers, such as Arduino and Raspberry Pi.

\section{A. Applications of Residential Sensors}

As predicted by Moore [14], while computer systems' processing power increases yearly, the cost of their components is reduced; this enables houses to be equipped with numerous connected sensors, which initially appeared in the market shortly after 2000 . These sensors should be low-cost and low-power to be easily deployed, maintained and upgraded on a mass scale.

One of the prevailing domains of smart home systems is security. To highlight the added value, we note that property damage accounted for $98.1 \%$ of insurance claims in 2018, according to the US Insurance Information Institute [15]. Low-power and low-cost sensor solutions can be used to prevent fires ( $\mathrm{CO}_{2}$ detection) and burglaries, in addition to monitoring buildings properties (e.g., moisture, temperature and corrosion of materials). Another domain is energy saving via monitoring the consumption of devices and trying to maintain thermal comfort. Moreover, the capabilities of smart home ecosystems are constantly upgrading. The following applications combine sensor devices:

- Smart lights: i.e., determination of the current flow to light bulbs.

- Smart sound devices: i.e., use of sounds (such as voice and clapping) to enable and disable processes.

- Smart thermostats: i.e., monitor of fridges and boilers.

- Smart meters: i.e., monitor of gas and electricity usage.

- Smart security cameras: i.e., trigger of applications' execution regarding the ventilation system to kitchen devices.

- Smart windows (switchable glasses): i.e., change of lighting of the windows' glass from translucent to transparent and vice versa, based on lighting conditions or opening/closing of windows.

It goes without saying that the key to enable these applications is the creation of interconnected wireless sensor networks (WSNs) using appropriate low-power communication technologies. In the following section, a selection of sensor types is analyzed further and for each category, low-cost and low-power sensory components are presented.

\section{B. Sensors for Smart Houses}

While there is a large number of residential sensors, not all of them make a house "smart". Specifically, a sensor is characterized as smart when it can process signals using a processing unit. This can be performed in multiple ways, such as memory-feedback, automatic calibration, accuracy-sensitivity error reduction and noise cancellation.

\section{Area Motion Detection}

Sensors can be utilized in smart houses to sense proximity and detect the motion of objects. These sensors are typically used in alarm systems to monitor rooms or spaces near windows and doors.

There are five types of area motion detection sensors: passive infra-red, microwave, ultrasonic, radar and vibration. The first type detects body heat (infrared energy) by monitoring changes in temperatures. The second type sends out microwave pulses and measures the reflections off of moving objects. Similarly, the third type measures distance using ultrasonic sound waves (non-audible by humans) shared and received to measure objects' proximate positions while radar types measure the reflection from a laser beam emission to the obstacles in an environment. Vibration motion sensors detect small vibrations that people cause when they move through a room; while they are small in size, they can be unreliable. None of these sensors measure motion per se. Last, dual motion sensors can combine multiple detection methods in an attempt to reduce false alarms. For example, it is not uncommon for a dual technology sensor to combine a passive infrared (PIR) sensor with a microwave sensor, each operating in a different spectrum. Nevertheless, any change in distance measurement will trigger rapid and reliable motion events [16] (Figure 1).

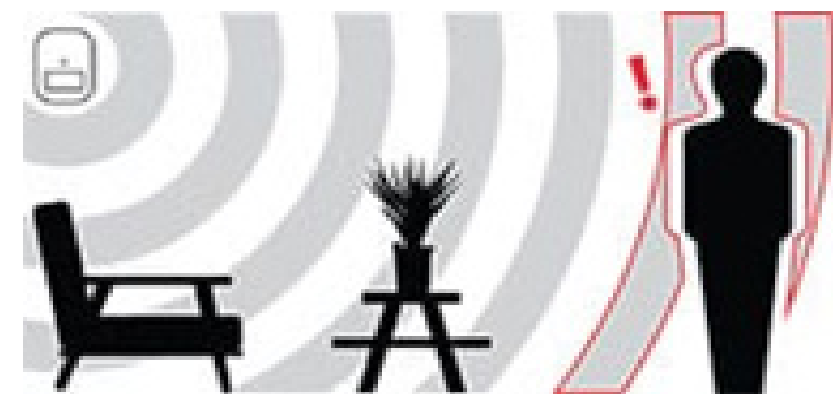

Figure 1: Dual-mode motion sensors [17].

Typical low-cost sensors used in smart home applications are HC-SR04, SEN14588 and VL6180.

\section{Flame-Gas Detection of Residential Sensors}

The demand for smart sensor applications in fire alarm systems is constantly growing. A flame detector is a sensor designed to detect and respond to the presence of a flame or fire. [18]: Optical flame detectors work by detecting the radiation (UV, IR, Visible and combinations) emitted at the instant of ignition. Photoelectric and ionization detectors are used for sensing slow and high burning fires. Specifically, the first uses a light sensing chamber which disrupt the pattern of a laser beam and thus triggers an alarm, when smoke enters the chamber. The second uses two electrically charged (ionized) plates which disrupts the flow of the current (ions), used to verify flame presence and quality.

The use of photoelectric sensing devices is preferable, as ionized sensors are highly sensitive and they can be triggered by steam (or even dust) [19]. Moreover, thermocouples are used extensively for monitoring flame presence in combustion heating systems and gas 
cookers. High levels of carbon dioxide $\left(\mathrm{CO}_{2}\right)$ may indicate a fire and can be detected by a carbon dioxide sensor.

While responses to a detected flame depend on the installation, they can include sounding an alarm, deactivating a fuel line (such as a propane or a natural gas line), and activating a fire suppression system (Figure 2).

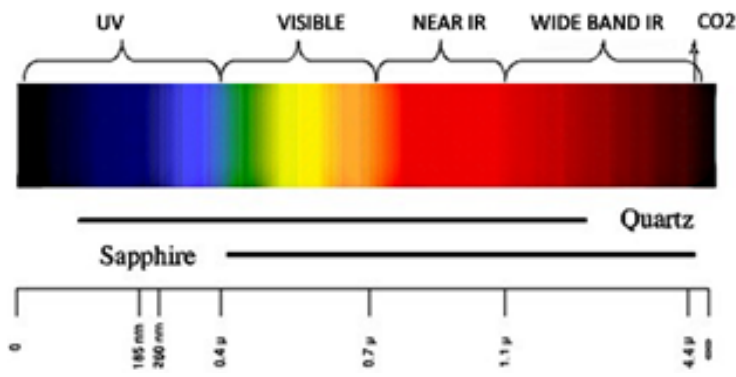

Figure 2: Flame detector type regions.

Typical low-cost sensors used in smart home applications are RE46C190 and YG1006.

\section{Toxic Gas Detection}

Besides fire smokes, gas leaks are among the main domestic dangers for residents. They constitute health hazards, especially when they are toxic and odorless. Toxic gas sensors are able to detect several gases, such as petroleum gas, smoke, alcohol, propane, hydrogen, methane and carbon monoxide. Their operation is the following: when the outer layer of a sensor is heated in high air temperatures, it absorbs oxygen from its surroundings. These sensors can determine the type of gas based on the oxygen's density, in addition to measuring the flow of the current [20]. However, the vast majority of low-cost toxic gas sensors are unreliable. Furthermore, they cannot distinguish between different gases but rather provide a generic estimate, based on a reference gas (Figure 3)

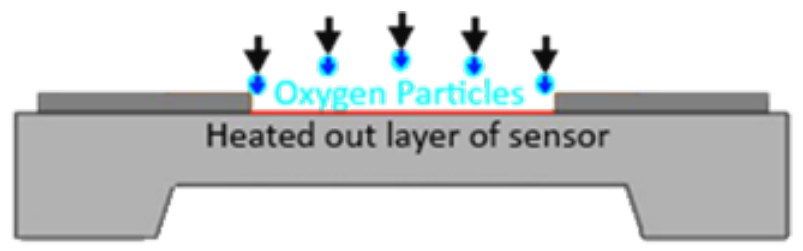

Figure 3: Flame detector type regions.

Examples of low-cost sensors used in smart homes applications are MQ2 and MQ135.

\section{Moisture-Leak Detection}

Another interesting category is moisture sensors which are typically used in agricultural industry applications. These sensors can also be utilized in homes to monitor possible leaks and moisture levels.

There are three types of humidity sensors: resistive, capacitive and thermal conductivity [21]. These sensors operate in the following way: resistive sensors use two pins (electrodes) that touch a sensor surface and between them, a middle layer is placed (substrate). When moisture (i.e. water vapor) occurs, the middle layer absorbs it, thus a change in resistance is measured, i.e. a difference in the electric flow.

Recent projects use capacitive sensors which measure the chargingdischarging time periods of capacitors, instead of resistance [22]. Thermal conductivity sensors measure humidity by calibrating the difference between the thermal conductivity of dry air and that of moist air. These sensors can measure either absolute or relative humidity. Resistive sensors are error prone, due to corrosion and dirt (Figure 4).

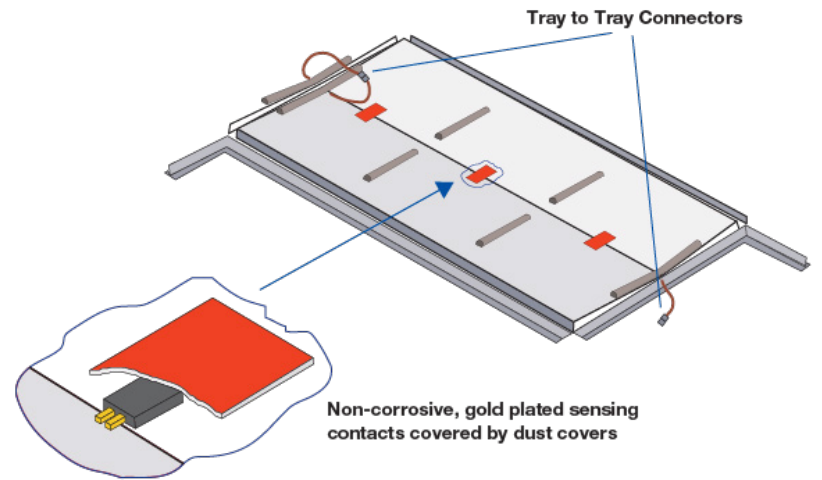

Figure 4: Ceiling tile water detector [23].

For instance, low-cost sensors used in smart homes applications which measure humidity and temperature are DHT11/DHT22, AM2320 and AHT20.

\section{Doors and Windows Security}

Contact sensors can serve as a tool for prevention of burglaries and home intrusions. These types of sensors consist of two connected magnets (typically opposite poles, but omnipolar solutions are also adopted) where a buzzer is activated, when they lose connection. Other solutions monitor magnetics fields via the Hall Effect, i.e. variations in the magnitude of magnetic fields $[24,25]$.

The Hall effect provides information regarding the type of magnetic pole and magnitude of the magnetic field. For example, a south pole would cause the device to produce a voltage output while a north pole would have no effect. Generally, Hall Effect sensors and switches are designed to be in "OFF", (open circuit condition) when there is no magnetic field present. They only turn to "ON", (closed circuit condition) when subjected to a magnetic field of sufficient strength and polarity (Figure 5).

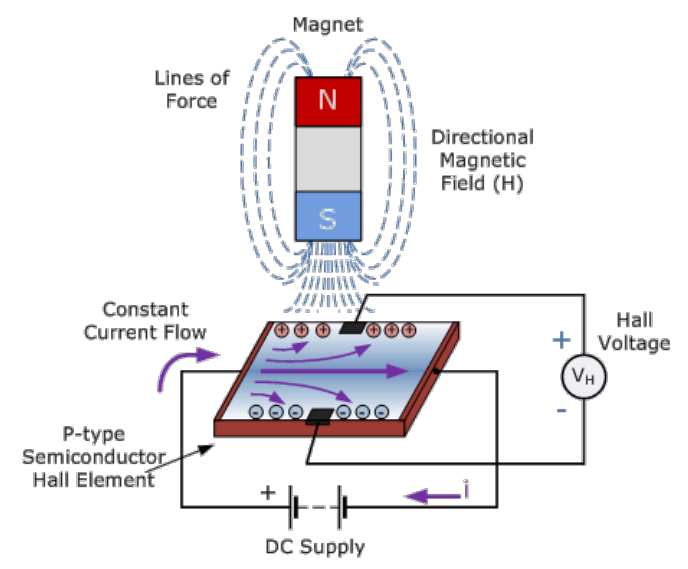

Figure 5: Hall Effect principles [26].

Typical low-cost sensory solutions used in smart homes applications regarding magnetic fields are 2SS52M and SS49E.

\section{Temperature Detection}

Sensors can also support residents with temperature monitoring. This is really important not only for safety reasons, but also to promote eco-friendly usage of heating or air-conditioning, by allowing users to adjust the thermostat.

There are four types of temperature sensors: thermocouples, Resistance Temperature Detectors (RTDs), thermistors, and semiconductorbased-ICs $[27,28]$. The principle of their operation is not similar to conventional thermometers which measure the expansion and contraction of a liquid (usually mercury or alcohol) in a sealed glass tube. Temperature sensors measure the resistance flow of the current where the change in temperature is correlated with the resistance 
value, based on the sensors' material. Typically, as temperature increases, most conductors also increase their resistance, as they are usually molted by metals [29]. Platinum is the most common material used to make RTDs while thermistors are usually made from a polymer or ceramic material. In most cases, thermistors are cheaper but they are also less accurate compared to RTDs. Thermocouples are made by joining two dissimilar metal wires together; the temperature difference between them produces a measurable voltage difference that is used to calculate the temperature (Seebeck effect). Lastly, semiconductor-based temperature sensors are ICs that measure their own die temperature by using the physical properties of a transistor (or an external transistor for remote ones) (Figure 6).

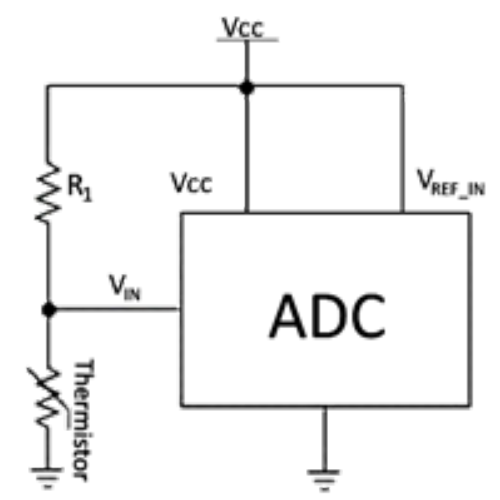

Figure 6: Typical thermistor interface.

For instance, low-cost sensors used in smart homes applications are LM35DZ, and TMP36. As previously stated, while there are sensors which measure both humidity and temperature, temperature sensors achieve higher accuracy measurements.

\section{Conclusion}

In this article, an analysis was provided regarding the core smart houses applications and some of the available sensors to support these systems. In all the examples mentioned, low-power and lowcost sensor solutions were proposed, since they are easy to install and enable the production of large sensor networks. All sensors' product manuals and datasets proposed can be found at [30]. Indeed, new methods for smart home automation and innovative solutions are constantly developed. Recent works focus on the connections between hardware and software products, compatibility within the software framework and their operation and user notification via cloud services processes. Thus, there is a need to shift the focus towards hardware and implement low-cost reliable solutions wherever these satisfy the application requirements. Residents are urged to invest in lowpower and low-cost residential sensors to ameliorate their everyday lives. This can be achieved affordably by connecting said sensors with electronic devices, such as Raspberry Pi and Arduino. Smart houses and wireless sensor networks are not just buzzwords; they are the future of residential sensing.

However, it should be made clear that these solutions only provide indicative information and not reliable knowledge about what is going on in the house and should be treated as such. Temperature and humidity sensors are extremely susceptible to thermal sources in the environment and often report higher values than those which exist in reality, while toxic gas sensors may provide vague or even random values. Consequently, while they are not appropriate solutions for the work place or industries where reliability plays a vital role as, they can be very useful in smart homes by capturing trends of the phenomena they monitor and providing early warning.

\section{References}

1. Miranda J, Mäkitalo N, Garcia-Alonso J, Berrocal J, Mikkonen T, et al.,
(2015) From the Internet of Things to the Internet of People. IEEE Internet Computing 19(2): 40-44.

2. Khan WZ, Rehman MH, Zangoti HM, Afzal MK, Armi N, et al., (2020) Industrial internet of things: Recent advances, enabling technologies and open challenges. Computers \& Electrical Engineering 81: 106522.

3. Alshouiliy K, Agrawal DP (2021) Confluence of 4G LTE, 5G, Fog, and Cloud Computing and Understanding Security Issues. Fog/Edge Computing For Security, Privacy, and Applications 2021, pp. 3-32.

4. Marr B (2018) How much data do we create every day? The mind-blowing stats everyone should read. Forbes, pp. 1-5.

5. Kuru K, Khan W (2020) A framework for the synergistic integration of fully autonomous ground vehicles with smart city. IEEE.

6. Sun Y, Song H, Jara AJ, Bie R (2016) Internet of things and big data analytics for smart and connected communities. IEEE 4: 766-773.

7. Atlam HF, Walters R, Wills G (2018) Internet of Things: state-of-the-art, challenges, applications, and open issues. (2018) International Journal of Intelligent Computing Research (IJICR) 9(3): 928-938.

8. Hassan QF, Khan AR, Madani SA (2017) Internet of things: Challenges, advances, and applications. CRC Press; 2017 Dec 15.

9. Gunge VS, Yalagi PS (2016) Smart home automation: a literature review. International Journal of Computer Applications 975:8887.

10. https://smartthings.developer.samsung.com.

11. https://github.com/alexa/avs-device-sdk.

12. https://developer.apple.com/homekit.

13. https://nest.com.

14. Moore GE (2006) Cramming more components onto integrated circuits, reprinted from electronics, volume 38, number 8, april 19, 1965, pp. $114 \mathrm{ff}$. IEEE solid-state circuits society newsletter 11(3): 33-35.

15. Insurance Information Institute. Facts and Statistics: Homeowners and renters insurance. https://www.iii.org/fact-statistic/facts-statisticshomeowners-and-renters-insurance.

16. Gazis A, Ioannou E, Katsiri E (2020) Examining the sensors that enable self-driving vehicles. IEEE Potentials 39(1): 46-51.

17. http://charlesjenningsassociates.com/services/security-control-systems/ motion-detection-systems.

18. http://www.microtech.co.th/pdf/flame-detector.pdf.

19. Hoefer U, Gutmachera D (2012) Fire gas detection. Procedia Engineering. 47: 1446-1459.

20. Durán A CM, Monsalve PA, Mosquera CJ (2016) Multisensor system for toxic gases detection generated on indoor environments. IOP Conference Series: Materials Science and Engineering, 2016 Nov 1 (Vol. 157, No. 1, p. 012029). IOP Publishing.

21. Yamazoe N, Shimizu Y (1986) Humidity sensors: principles and applications. Sensors and Actuators 10(3-4): 379-398.

22. Sehrawat D, Gill NS (2019) Smart sensors: Analysis of different types of IoT sensors. 3rd International Conference on Trends in Electronics and Informatics (ICOEI), pp. 523-528.

23. https://www.wateralert.com/ceiling-guard-standard-series.

24. Lenz J, Edelstein S (2006) Magnetic sensors and their applications. IEEE Sensors journal. 6(3): 631-649.

25. Ripka P, Janosek M (2010) Advances in magnetic field sensors. IEEE Sensors journal. 10(6): 1108-1116.

26. Castaño Sánchez M (2015) Design of a position encoder using infrared sensors. Report. Polytechnic University of Catalonia Faculty of Electrical Engineering and Information Technology.

27. Mohan C Jha (2015) Thermal Sensors. Principles and applications for semiconductor industries. Springer.

28. McGee TD (1988) Principles and methods of temperature measurement. 
John Wiley \& Sons.

29. Liu C, Ren W, Zhang B, Lv C (2011) The application of soil temperature measurement by LM35 temperature sensors. IEEE International Conference on Electronic \& Mechanical Engineering \& Information

\section{Technology. pp. 1825-1828.}

30. https://github.com/AlexandrosGazis/IoT-Sensors-for-Smart-HomesDatasheets. 